\title{
A Feasibility Study on Seasonal Changes in Wrist Pulse using Cumulative Periodogram
}

\author{
Jang-Han Bae, Jaeuk U. Kim \\ Korea Institute of Oriental Medicine \\ 1672 Yuseong-daero, Yuseong-gu, Daejeon, Korea \\ fcbest11@kiom.re.kr; jaeukkim@kiom.re.kr
}

\section{Extended Abstract}

Wrist pulse signal acquired from the radial artery is considered to be affected by different environmental factors such as seasonality. Although, seasonal changes of wrist pulse characteristics have been reported with descriptive comments in ancient literatures of traditional Eastern medicine, clinical studies or their scientific verifications with pulse waveform analysis were rarely done. Indeed, most prior studies of the seasonality were just focused on the relationship between blood pressure and environmental temperature variations, showing increased values in the winter compared to the summer [1]. Therefore, different points of view is needed for studying the seasonal adaptation of wrist pulse signal. Pulse analysis in the frequency domain can contain certain physiological and pathological conditions [2]. Fast Fourier transform (FFT) for observing peak frequencies was generally used and power spectral density (PSD) of certain frequency band was calculated to determine the differences between different subject groups [3]. However, fluctuations in the PSD derived from the calculation of FFT and very nature data inevitably interrupt the generation of fine-structure features of the spectra [4]. Therefore, appropriate approach to overcome the limits of the PSD analysis is highly needed to observe the seasonality of wrist pulse in frequency domain. The cumulative spectral power (CSP) describes the distribution of power below a certain frequency. It does not suffer the subtlety and automatically induces some smoothing which can see in the cumulative periodogram $(\mathrm{CP})$. It has been also known that cardiovascular disorder can be detected using CSP analysis unambiguously [4]. However, pulse analysis using CSP for observing the differences among the four seasons was never reported before. This feasibility study suggests a simple graphical method using CP for examining the seasonal change in wrist pulse.

Healthy 32-year-old man and 28-year-old woman were enrolled in this study. Left wrist pulse signals were measured eight times with one-week interval for each season. Definition of each four seasons is as follows; spring (April-May), summer (July-August), autumn (October-November), and winter (January-February). Measurement of fasted subjects began at 10 a.m. using pulse tonometric device developed at the Korea Institute of Oriental Medicine [5] after 20 minutes

relaxation in controlled room with $24^{\circ} \mathrm{C}$. After Pre-processing with noise and baseline wander removal, the pulse measured for 60 seconds with constant contact pressure was used for cumulative spectral analysis. To observe the shift in power of each season, CSP within $0 \sim 10 \mathrm{~Hz}$ of each season was calculated and CPs were plotted. To detect the shape difference of underlying spectra, normalized CPs between the two out of the four seasons were also plotted. As a results, pulse signals acquired in the winter have significant differences in energy distribution with low CSP. In addition, there is a strong implication that the power of pulse signal acquired in the winter shifts from higher to lower frequencies with accompanying increased dispersion compared to the other three seasons. In conclusion, distinctive information of cardiovascular activities in the winter was detected in frequency domain and new approaches with CSP could be useful tool for pulse waveform analysis. Large population-based clinical study will be needed for more confirmative results. 


\section{References}

[1] P. A. Modesti, "Season, temperature and blood pressure: A complex interaction," European Journal of Internal Medicine., vol. 24, pp. 604-607, 2013.

[2] L. Xu, M. Q. H. Meng, C. Shi, K. Wang and N. Li, "Quantitative analyses of pulse images in traditional Chinese medicine," Med. Acupunct., vol. 20, no. 3, pp. 175-189, 2008.

[3] Y. T. Liao, H. Y. Chen, C. M. Huang, M. Ho, J. G. Lin, C. C. Chiu and F. J. Chen, "The pulse spectrum analysis at three stages of pregnancy," J. Altern. Complement. Med., vol. 18, no. 4, pp. 382-386, 2012.

[4] S. C. Lee and R. D. Peters, "A new look at an old tool - the cumulative spectral power of fast-Fourier transform analysis," Dept. Physics., Mercer Univ., Macon, GA 31207, 2009, [Online]. Available: http://arxiv.org/abs/0901.3708

[5] J. H. Bae, Y. J. Jeon, J. Y. Kim, and J. U. Kim. "New assessment model of pulse depth based on sensor displacement in pulse diagnostic devices," Evid Based Complement Alternat Med., 938641, 2013. 